\title{
Feedforward, Cascade and Model Predictive Control Algorithms for De-Oiling Hydrocyclones: Simulation Study
}

\author{
Mishiga Vallabhan $\mathrm{K} \mathrm{G}^{1}$ Jose Matias $^{2}$ Christian Holden ${ }^{1}$ \\ ${ }^{1}$ Department of Mechanical and Industrial Engineering, Norwegian University of Science and Technology, N-7491 \\ Trondheim, Norway. E-mail:\{mishiga.vallabhan,christian.holden\}@ntnu.no \\ ${ }^{2}$ Department of Chemical Engineering, Norwegian University of Science and Technology, N-7491 Trondheim, Nor- \\ way. E-mail: \{jose.o.a.matias\} @ntnu.no
}

\begin{abstract}
Maintaining the efficiency of the produced-water treatment system is important for the oil and gas industry, especially taking into consideration the environmental impact caused of the produced-water. De-oiling hydrocyclones are one of the most common type of equipment used in the produced-water treatment system. The low residence time of this device makes it difficult for a control system to maintain efficiencies at different plant disturbances. In this paper, a control-oriented hydrocyclone model with a traditional pressure drop ratio (PDR) controller is analysed, and the inability of the PDR controller to maintain the efficiency when increasing the inlet concentration is shown experimentally as well as in simulation. Then, we propose three control schemes for dealing with this issue: a feed-forward, a feed-back/cascade and a model predictive controller. We show in simulation that all proposed schemes are able to improve and maintain the efficiency of hydrocyclones considering the upstream disturbances, such as variations in inlet oil concentrations and inflow rates. We also discuss the characteristics of the three methods and propose guidelines for choosing the appropriate scheme based on the available resources at the industrial site (such as measurements, hardware and software at hand).
\end{abstract}

Keywords: De-oiling hydrocyclones, Control Schemes, Simulation

\section{Introduction}

The treatment of produced-water (naturally occurring water that comes out of the ground along with oil and water) is an inevitable process in oil and gas production facilities. In Norway, for example, nearly $160 \times 10^{6} \mathrm{~m}^{3}$ of this waste stream are discharged annually, which corresponds to a potential release of 1900 tonnes of crude oil to sea (Beyer et al., 2019). Traditionally, the produced-water is re-injected into the well. However, the constant increase of the wastewater volumes and the associated costs make this choice debatable. On the other hand, the water can be discharged into the sea if the oil is separated beforehand. Processes such as membrane filtration, compact flotation units and separation through hydrocyclones can be used for this end. The challenge then becomes guaranteeing that they operate efficiently, complying with the requirements set out in local regulations.

Among the disposal alternatives, hydrocyclones are specially suited for offshore sites. They are compact, light, and do not require any additional chemicals or gases to be injected for the operation. Moreover, they have already been successfully implemented in subsea 
below $800 \mathrm{~m}$ (Orlowski et al., 2012). The main problem with this alternative is the hydrocyclones low residence times, which makes them more susceptible to upstream variations such as frequent changes in inlet oil concentration, inflow rate, etc. Typically, control schemes that use pressure drop ratio (PDR) to control the separation are implemented to reject these disturbances. Since this is an indirect way of controlling the hydrocyclones, this option can reduce their efficiency significantly and result in violations of the environmental requirements.

In this paper, we propose three new control schemes that take into consideration disturbances such as inlet oil concentration and inflow rate. Two of the proposed approaches, one based on feed-forward and another on feedback/cascade schemes, automatically adjust the setpoint of the PDR controller based on the current disturbance. The third one uses model predictive control to minimize the variation of underflow concentration from a reference value. We also discuss the pros and cons of the alternatives and provide an assessment of the possible implementation issues.

\subsection{Previous work}

The control aspects of hydrocyclones have been gaining more and more focus in recent years. A controloriented approach based on transfer functions models using experimental data from a test rig was developed by Durdevic et al. (2015). Then, a grey box static model to calculate the separation efficiency of hydrocyclones based on flow resistance and droplet trajectory was developed by Bram et al. (2018). A control-oriented model for de-oiling hydrocyclone with a swirl element was developed by Das and Jäschke (2018). In Bram et al. (2020), a virtual flow resistance model with an extended trajectory model was developed and performance of hydrocyclone was compared using the model and experimental data from a scaled pilot plant. A first-principles model for de-oiling hydrocyclones based on pressure-flow relationship, separation efficiency and dynamic mass balance was developed by Vallabhan K G et al. (2020).

Husveg et al. (2007) studied the performance of hydrocyclones to varying inflow rates and emphasised that adequate operational control of hydrocyclone is necessary to maintain efficiency. Considering a first stage gravity separator and a hydrocyclone as a single plant, a robust control strategy was proposed by Durdevic and Yang (2018) and a model predictive control was proposed by Hansen et al. (2018). Later, nonlinear model based control algorithms to improve the efficiency of hydrocyclones were proposed by Vallabhan and Holden (2020).

When the hydrocyclones are connected to bulky first stage gravity separators, the rate of change of disturbances at the inlet of hydrocyclones are expected to be less frequent and of relatively low amplitude. However, when we move towards compact separation at subsea, e.g., Marlim fields in Brazil (Pereira et al., 2012), pipe separators are used upstream of the hydrocyclones. Due to the compactness of this type of separators, the hydrocyclones will be subjected to frequent changes in inlet oil concentration, oil droplet distributions, inflow rates etc. Consequently, the dynamics are faster and difficult to control. The existing control schemes for hydrocyclones uses an indirect pressure drop ratio to control the separation of hydrocyclones. PDR control scheme works well if the disturbance is in the inflow rate of the hydrocyclones as it gets reflected in the pressure drop. However, the PDR control scheme cannot detect the changes such as inlet oil concentration and inlet oil distribution. Hence, it is necessary to address these variations when we are designing a control system for hydrocyclones (produced-water treatment) subsea.

\section{Hydrocyclone model}

This section gives a brief description of the hydrocyclone model that is used as the "true" system for studying the different control schemes proposed in this paper. The model is based on Vallabhan $\mathrm{K}$ G et al. (2020). A simplified diagram of a hydrocyclone liner and a block diagram representation of a controloriented model for hydrocyclone is shown in Figure 1.

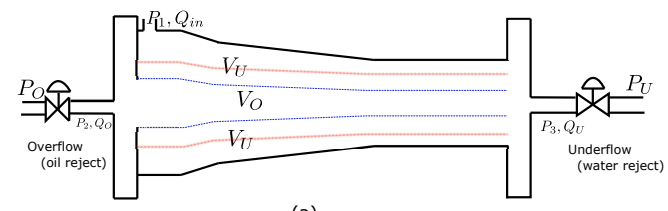

(a)

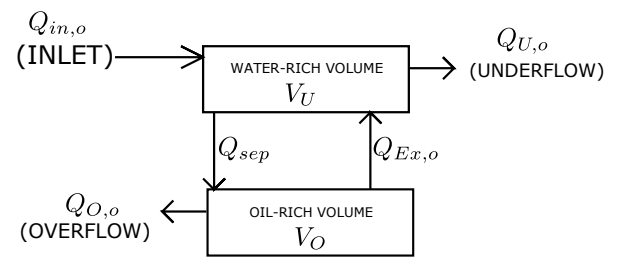

(b)

Figure 1: Simplified diagram of a hydrocyclone liner representing the 'oil-rich volume' $\left(V_{O}\right)$ and the 'water-rich volume' $\left(V_{U}\right)$ (shown in (a)) and a block diagram representation of a control-oriented model making use $\left(V_{O}\right)$ and $\left(V_{U}\right)$ (shown in $(\mathrm{b})$ ) 
We divide the hydrocyclone liners into two volumes: one is the 'oil-rich volume' $\left(V_{O}\right)$ and the other is the 'water-rich volume' $\left(V_{U}\right)$. The oil droplets entering the reverse-flow zone (between the red lines in Figure 1) go to the oil-rich volume and leave the system at the overflow outlet. The remaining oil in the water-rich volume comes out at the underflow outlet. A simple mass bass balance formulation is used to model the inflow and outflow of the oil inside the volumes. Since the oil density is assumed constant, we write the mass balances as

$$
\begin{aligned}
& \frac{d V_{O, o}}{d t}=Q_{s e p}-Q_{O, o}-Q_{E x, o}, \\
& \frac{d V_{U, o}}{d t}=Q_{i n, o}-Q_{s e p}-Q_{U, o}+Q_{E x, o} .
\end{aligned}
$$

where $V_{O, o}$ is the volume of oil in the oil-rich volume $V_{O}$ $; V_{U, o}$ is the volume of the oil in the water-rich volume $V_{U} ; Q_{\text {sep }}$ is the flowrate of the separated oil entering $V_{O} ; Q_{O, o}$ is the flowrate of oil at the overflow; $Q_{E x, o}$ is the excess flowrate of oil entering $V_{U}$ (which is the case when the overflow opening is small and $V_{O}$ is filled with oil); $Q_{i n, o}$ is the inflow rate of oil; and $Q_{U, o}$ is the flowrate of oil at the underflow. Here all the volumes are expressed in $\left[\mathrm{m}^{3}\right]$ and flowrates in $\left[\mathrm{m}^{3} / \mathrm{h}\right]$. The parameters of hydrocyclone liners used in this paper is the same as in Vallabhan K G et al. (2020).

The internal separation is expressed in terms of volumetric flow and given as $\frac{Q_{s e p}}{Q_{i n, o}}$. In Vallabhan K G et al. (2020), a droplet trajectory analysis is used to calculate the internal separation. Oil droplets of different size categories are tracked using their axial, tangential and radial velocity components. If the droplets cross the reverse-flow zone boundary (shown in red in Figure 1) inside the hydrocyclone liner, they are assumed to be separated. The authors use a polynomial approximation to compute the internal separation $\frac{Q_{s e p}}{Q_{i n, o}}$. In this work, we use the same polynomial approximation model to calculate the internal separation under the assumption that we know the inflow rate and its value is between $1.5 \mathrm{~m}^{3} / \mathrm{h}$ and $3.5 \mathrm{~m}^{3} / \mathrm{h}$. This approximation for internal separation is given as:

$$
\frac{Q_{\text {sep }}}{Q_{\text {in }, o}}=p_{2} Q_{O}^{2}+p_{1} Q_{O}+p_{0} .
$$

where $p_{2}=-9.447 \mathrm{e} 7, p_{1}=9024, p_{0}=0.7648$. Here, the other factors, such as underflow rate $Q_{U}$, that affect the separation are kept constant and, hence,the separation is assumed to be only a function of overflow rate $Q_{O}$.

The excess oil entering $V_{U}$ is computed as:

$$
Q_{E x, o}= \begin{cases}Q_{s e p}-Q_{O}, & \text { if } Q_{s e p}-Q_{O}>0 \\ 0, & \text { otherwise }\end{cases}
$$

The volume fractions of oil in the two volumes $V_{O}$ and $V_{U}$ are defined as:

$$
\beta_{O, o}=\frac{V_{O, o}}{V_{O}}, \quad \beta_{U, o}=\frac{V_{U, o}}{V_{O}} .
$$

For simplicity, we assume that the internal volumes $V_{O}$ and $V_{U}$ are well mixed, which implies that the compositions in the outflows $Q_{O}$ and $Q_{U}$ are the same as the internal compositions. Then, we get from the definition of Eq. (4):

$$
Q_{O, o}=\beta_{O, o} Q_{O}, \quad Q_{U, o}=\beta_{U, o} Q_{U} .
$$

Re-writing (1) in terms of volume fractions gives

$$
\begin{aligned}
& \frac{d \beta_{O, o}}{d t}=\frac{1}{V_{O}}\left(Q_{s e p}-\beta_{O, o} Q_{O}-Q_{E x, o}\right), \\
& \frac{d \beta_{U, o}}{d t}=\frac{1}{V_{F}}\left(Q_{i n, o}-Q_{s e p}-\beta_{U, o} Q_{U}+Q_{E x, o}\right) .
\end{aligned}
$$

The two outflow $Q_{O}$ and $Q_{U}$ are calculated based on the simple valve equations given as:

$$
\begin{aligned}
& Q_{U}=C_{v 1} Z_{U} \sqrt{\frac{2\left(P_{3}-P_{U}\right)}{\rho_{U}}} \\
& Q_{O}=C_{v 2} Z_{O} \sqrt{\frac{2\left(P_{2}-P_{O}\right)}{\rho_{O}}},
\end{aligned}
$$

where, $C_{v 1}$ and $C_{v 2}$ are the valve constants of the underflow and overflow valves, $P_{2}$ is the pressure at the overflow outlet, $P_{3}$ is the pressure at the underflow outlet, $Z_{U} \in[0,1]$ and $Z_{O} \in[0,1]$ are the valve positions, and $\rho_{U}$ and $\rho_{O}$ are the densities of liquid at the underflow and overflow outlets, respectively. $P_{O}$ is the downstream pressure of the overflow valve, and $P_{U}$ is the downstream pressure of the underflow valve . In this paper, we assume that $P_{O}$ and $P_{U}$ are known and equal to the atmospheric pressure. The pressure $P_{2}$ and $P_{3}$ are assumed to be either measured or can be calculated based on the pressure-flow model, as in Vallabhan K G et al. (2020).

\section{Typical control strategy of hydrocyclones}

The typical operational control of hydrocyclone consists of two control loops, the first one is flow rate control and the second one is flow split control Husveg et al. (2007). Figure 2 shows a simplified P\&ID representing the two control loops.

The goal of the flow rate control loop is to maintain a certain level in the upstream tank separator and thereby maintaining the inflow rate of hydrocyclone between $Q_{i n, \min }$ and $Q_{i n, \max }$. This inlet flowrate range 


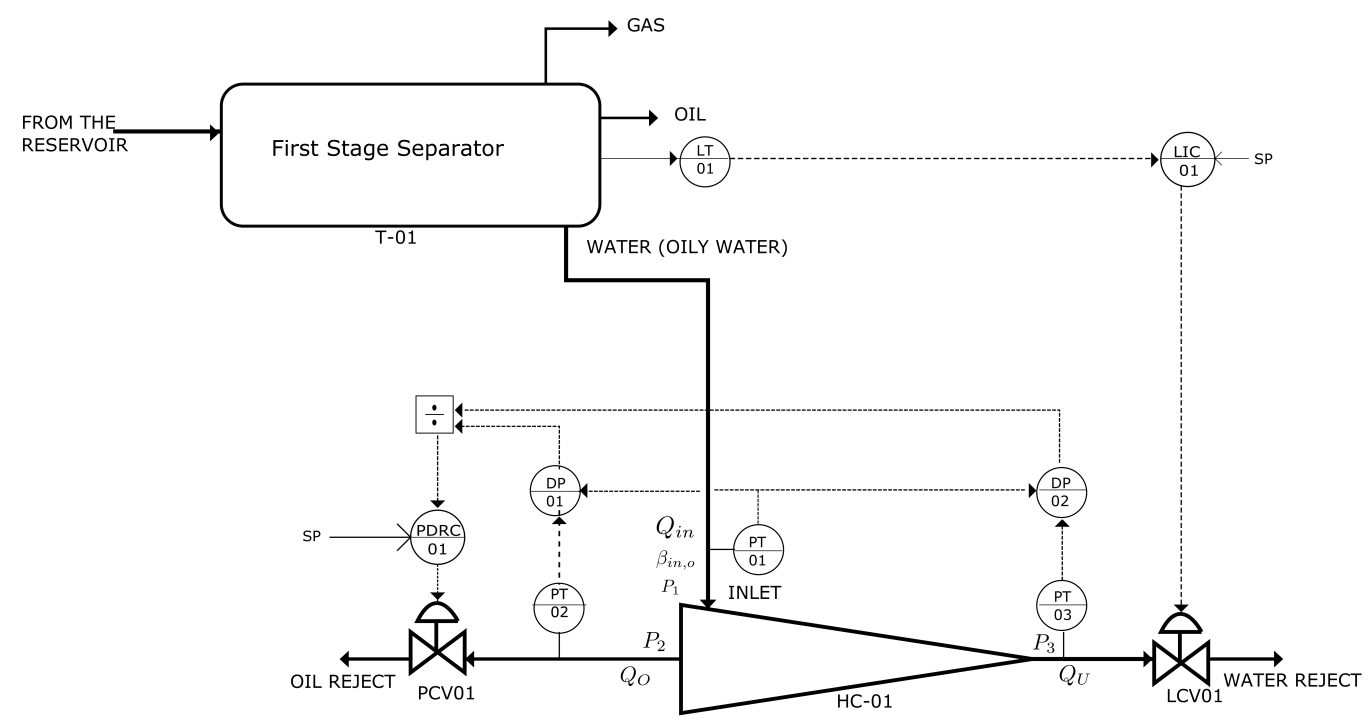

Figure 2: A typical control scheme of hydrocyclone representing flow rate and flow split control

characterizes an efficiency plateau, in which the oilwater separation is more effective (see Figure 3). This is achieved by manipulating the underflow control valve LCV01. In turn, the flow split control aims at maintaining a sufficient overflow rate in the hydrocyclone. The objective is to have good separation and maintain efficiency. The overflow control valve PCV01 is adjusted to keep a sufficient flow split ratio $F_{s}$, where $F_{s}=\frac{Q_{O}}{Q_{i n}}$.

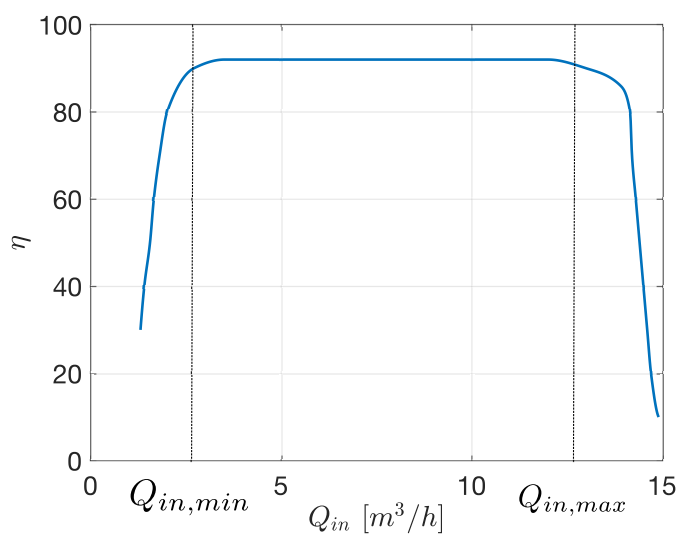

Figure 3: Relationship between efficiency and inflow rate of a hydrocyclone (Husveg et al., 2007). The efficiency of hydrocyclone is defined as $\eta=1-\frac{\beta_{U, o}}{\beta_{i n, o}}$, where $\beta_{i n, o}$ is the volume fraction of oil at the hydrocyclone inlet and $\beta_{U, o}$ is the volume fraction of the oil at the underflow outlet (water reject).

Since the flow split has linear relationship with pressure drop ratio (PDR) and pressure sensors are cheaper and more reliable than flow sensors, PDR is given as the setpoint to controller PDRC01 instead of the flow split. The pressure difference at the inlet and the two outlets is used to calculate the pressure drop ratio (PDR), defined as:

$$
\mathrm{PDR}=\frac{P_{1}-P_{2}}{P_{1}-P_{3}}
$$

where $P_{1}, P_{2}, P_{3}$ are the pressures at the inlet, overflow and underflow, respectively.

\subsection{Issues with the typical control strategy}

The experimental results in Meldrum (1988) show that PDR in the range 1.5 to 3 maintains the efficiency of the separation, if the inflow rate is kept at the efficiency plateau. However, keeping the setpoint of the PDR controller constant during plant disturbances (e.g., changes in the inlet oil concentration) can reduce the efficiency of hydrocyclones (Meldrum, 1988). Such changes may not be frequent in systems where first stage separators are gravity based with sufficient buffer volumes. On the other hand, if compact separators are used instead, frequent changes in the inlet oil concentrations for hydrocyclones are likely to happen.

In order to illustrate the effect of constant setpoint to the PDR controller of a hydrocyclone, we simulate the model described in Section 2 with a simple PI controller (which acts as a PDR controller). Here, the process variable is PDR, which is calculated based on (7), and the manipulated variable is the overflow rate $Q_{O}$. We assume that the underflow valve is kept at a constant opening, which maintains the inflow rate 
in the efficiency plateau. The simulation results are shown in Figure 4.
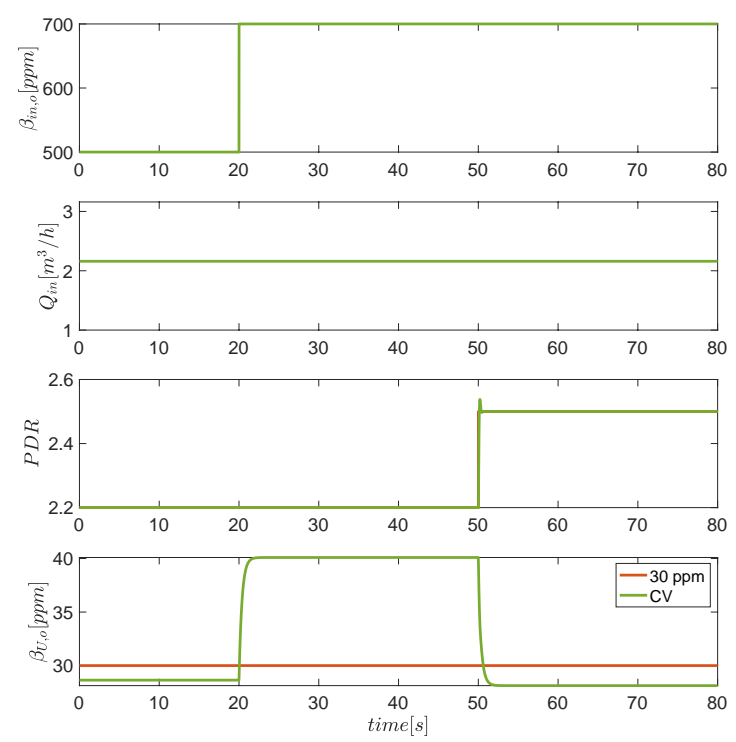

Figure 4: Simulation result showing increase in the $\beta_{U, o}$ with increase in $\beta_{\text {in }}$ when fixed PDR setpoint. At $50 \mathrm{~s}$, the PDR setpoint is manually adjusted to reduce $\beta_{U, o}$ below $30 \mathrm{ppm}$.

We start the simulation at steady-state with a PDR setpoint of 2.2 , inlet oil concentration $\beta_{i n, o}$ at $500 \mathrm{ppm}$ and inflow rate of $2.2 \mathrm{~m}^{3} / \mathrm{h}$. With this operating condition, the PDR setpoint of 2.2 keeps the underflow oil concentration, $\beta_{U, o}$, below $30 \mathrm{ppm}$. At $20 \mathrm{~s}, \beta_{\text {in,o }}$ is increased from $500 \mathrm{ppm}$ to $700 \mathrm{ppm}$. The change in $\beta_{i n, o}$ increases the underflow oil concentration, which decreases the efficiency of hydrocyclones. Since the inflow rate remains the same, the PDR does not change and the controller cannot react to the increase in the inlet oil concentration. At $50 \mathrm{~s}$, the setpoint of the PDR controller is increased manually to bring the $\beta_{U, o}$ below $30 \mathrm{ppm}$. The simulation results indicate that the effect of changes in inlet oil concentration should be taken into consideration while designing control system for hydrocyclones.

We also did a laboratory experiment at a test rig to show the effect of constant PDR on increase in inlet oil concentration. The control loop implemented at the experimental setup is similar to the P\&ID shown in Figure 2. The underflow valve is LCV01 is kept at $90 \%$ and PCV01 is controlled by the PRDC01. The system is started with an inlet oil concentration of $350 \mathrm{ppm}$. In order to keep the underflow oil concentration below $30 \mathrm{ppm}$, the setpoint of the PDR controller is defined as 1.5. Later, the inlet oil concentration is increased to $800 \mathrm{ppm}$ without changing the inflow rate. In Figure 5, we can see that the PDR is not changed and hence the controller PDRC01, does not take any action. However, we can see that the underflow oil concentration increases when the inlet oil concentration increases and this reduces the efficiency of hydrocyclone, confirming the simulation results. In order to deal with this problem, we propose three different control schemes. A feed-forward controller, a feedback/cascade controller, and also a model predictive controller. We compare their performance using the simulation scenario of Figure 4. After presenting the three methods, we discuss the advantages and challenges with their implementation.
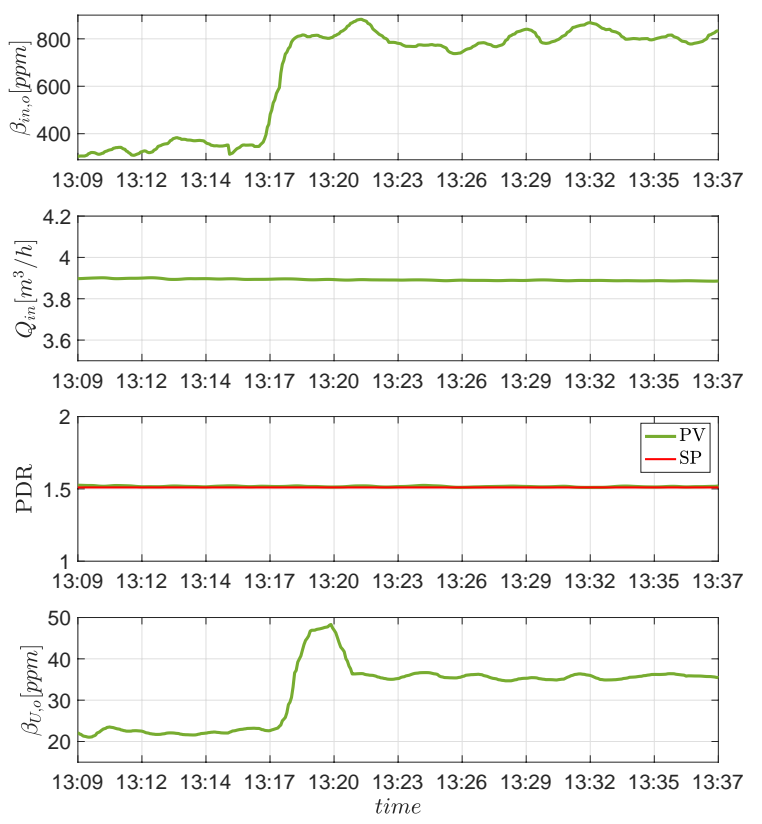

Figure 5: Laboratory experiment showing increase in the $\beta_{U, o}$ with increase in $\beta_{\text {in }}$ when fixed PDR setpoint.

\section{Feed-forward algorithm for hydrocyclones}

If the disturbance can be measured before entering the system, then feed-forward control can be used for disturbance compensation. In this control scheme, a model is used to adjust the setpoints automatically based on the certain measurements. For example, in the system shown in Figure 4, we can use the measurements of the inlet-oil concentration $\beta_{i n, o}$ and the inflow rate $Q_{i n}$ to adjust the PDR controller setpoint. By using this feed-forward scheme, we guarantee that the underflow oil concentration remains below 30 ppm without the need of a manual intervention. The block diagram of the implemented feed-forward control scheme 


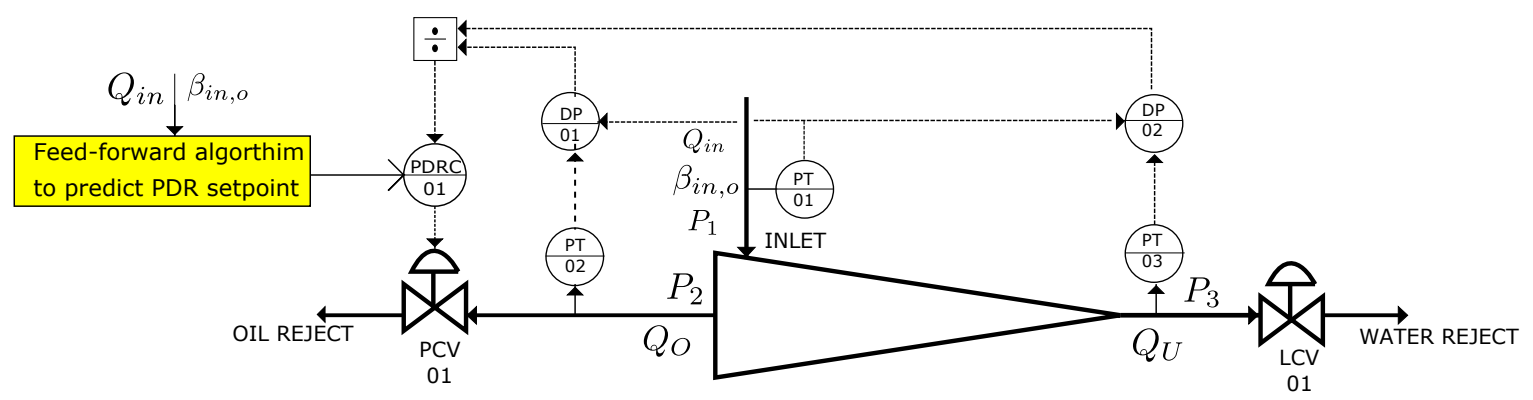

Figure 6: A diagrammatic representation of the feed-forward approach adjusting the PDR setpoint

is shown in Figure 6. For the feed-forward model, we use a Gaussian process regression (GPR) (MATLAB, 2021) model to predict the setpoints of the PDR controller based on the inflow rate and the inlet oil concentration. Hence, the GPR model has two input variables, $Q_{i n}$ and $\beta_{i n, o}$, and one response variable, the desired PDR setpoint. We use GPR models because they are relatively easier to handle and interpret than conventional machine learning methods, such as neural networks (Williams and Rasmussen, 2006). However, one can use simple linear regression models for instance, given that they yield accurate predictions.

The training data for the GPR model identification is generated by simulating the model described in the Section 2 with a PDR controller. Then, inflow rates are varied from $1.8 \mathrm{~m}^{3} / \mathrm{h}$ to $2.8 \mathrm{~m}^{3} / \mathrm{h}$, inlet oil concentration is varied from $500 \mathrm{ppm}$ to $1000 \mathrm{ppm}$, and PDR setpoint is manually varied from 2 to 3.5 . Later, the PDR values that keep the $\beta_{U, o}$ below $30 \mathrm{ppm}$ are filtered out. In an industrial setup, instead of using simulations, one can use historical data for inlet oil concentration, inflow rate and the changes in the PDR setpoint performed by the operator.

The data set is then fed to the MATLAB function fitrgp. The fitrgp function returns a GPR model.

Later, the model can be imported as a function in MATLAB and used as the feed-forward block in the control scheme shown in Fig. 6. Since this is a databased model, its extrapolation capacity is poor. The model predictions are valid for the flow rate range $1.8 \mathrm{~m}^{3} / \mathrm{h}$ to $2.8 \mathrm{~m}^{3} / \mathrm{h}$ and the inlet oil concentration $500 \mathrm{ppm}$ to $1000 \mathrm{ppm}$, which encloses the considered operating region. If a new region is to be considered, a new GPR model needs to be generated.

\subsection{Simulation results}

Figure 7 shows the simulation results using the feedforward model (GPR model) to predict the setpoint changes of the PDR controller. We plot the variations of inlet oil concentration $\beta_{i n, o}$, PDR setpoint predicted by the feed-forward model, inflow rate $Q_{i n}$, and con- centration of the oil at the underflow outlet $\beta_{U, o}$. For representing the real system, we use the hydrocyclone model described in Section 2. We start the simulation with inflow rate $2.2 \mathrm{~m}^{3} / \mathrm{h}$ and the inlet concentration of $500 \mathrm{ppm}$. Then at $50 \mathrm{~s}$, we increase the inlet concentration to $700 \mathrm{ppm}$. The feed-forward model detects the change in the inlet oil concentration and predicts the setpoint to be 2.8. Thus, the controller is able to maintain the underflow oil concentration below $30 \mathrm{ppm}$. As an additional check to the GPR model, we increase the inflow rate to $2.6 \mathrm{~m}^{3} / \mathrm{h}$ at $100 \mathrm{~s}$. The feed-forward model changes the controller setpoint to 2.2 , thereby keeping $\beta_{U, o}$ below $30 \mathrm{ppm}$. The PDR controller parameters are given in Table 2.
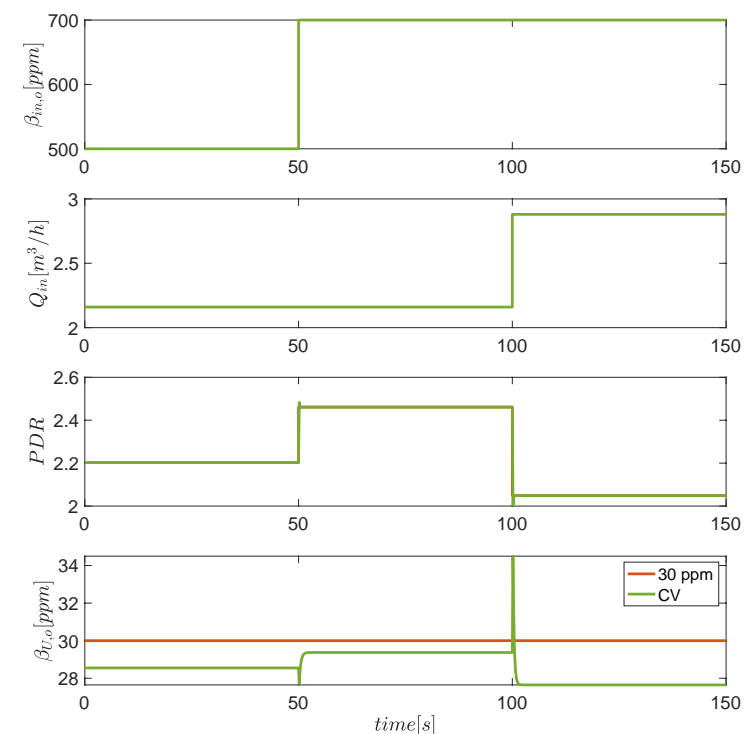

Figure 7: Simulation results showing the feed-forward control scheme. Here, the inlet oil concentration changes at $50 \mathrm{~s}$ and the inflow rate is changed at $100 \mathrm{~s}$. 


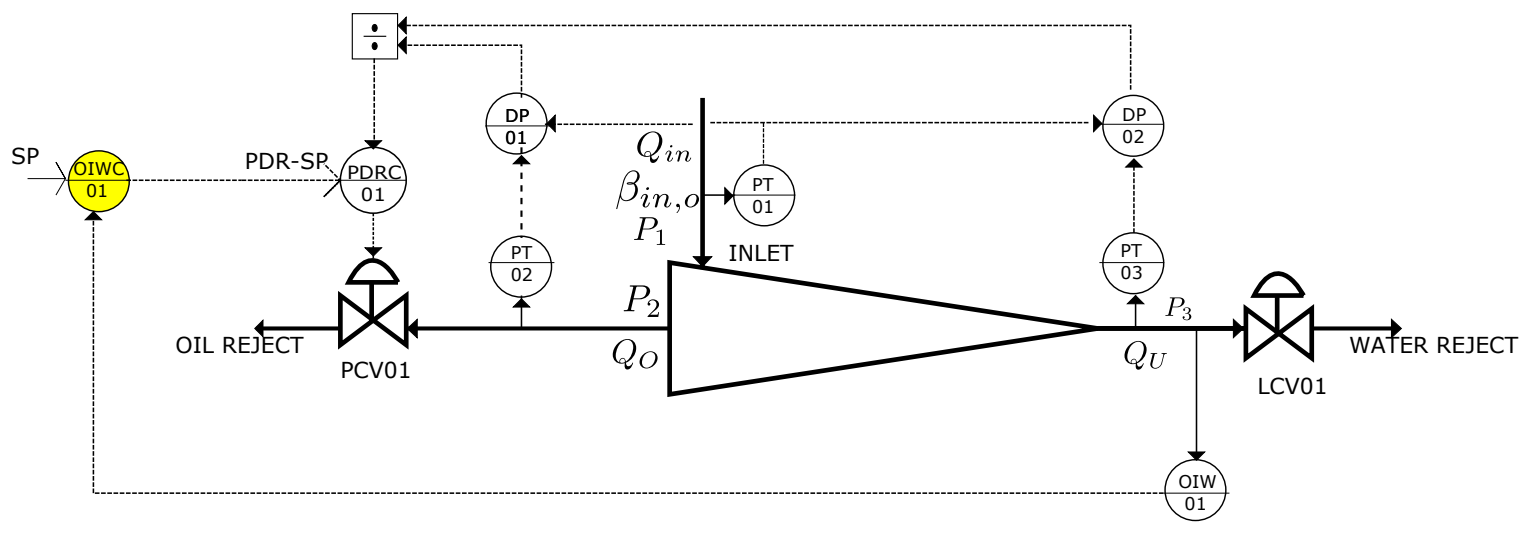

Figure 8: A diagrammatic representation of the cascade approach adjusting the PDR setpoint

\section{Feedback/cascade control for hydrocyclones}

As before, the goal of the feedback/cascade approach is to adjust the PDR controller setpoints automatically. For its implementation, the only pre-requisite is a sensor that measures the oil concentration at the underflow outlet with a sampling rate matching the control system. The diagrammatic representation of the feedback approach is shown in Figure 8. Here, a simple PI controller, OIWC01 acts as the primary controller. We can set the desired underflow concentration of oil as a setpoint to the primary controller. Later, this primary controller adjusts the setpoint of the secondary controller PDRC01 and maintains the underflow oil concentration $\beta_{U, o}$ to a desired level.

\subsection{Simulation results}

Again, the model of Section 2 is used for representing the real system. Figure 9 shows the simulation results. We repeat the scenario from the feed-forward algorithm analysis. We start the simulation with inflow rate of $2.2 \mathrm{~m}^{3} / \mathrm{h}$ and the inlet oil concentration of $500 \mathrm{ppm}$. The setpoint of the primary controller is kept at $30 \mathrm{ppm}$. At $50 \mathrm{~s}$, the inlet oil concentration is increased to $700 \mathrm{ppm}$, which increases $\beta_{U, o}$. The primary controller automatically adjusts the setpoint of PDRC01 to increase the separation and bring down $\beta_{U, o}$ to $30 \mathrm{ppm}$. Later, at $200 \mathrm{~s}$, we increase the inflow rate to $2.6 \mathrm{~m}^{3} / \mathrm{h}$. Even though it improves the separation, the primary controller tracks the setpoint of $30 \mathrm{ppm}$ and, hence, adjusts the setpoint of PDRC01. The controller parameters of the primary and secondary controller are given in Table 2
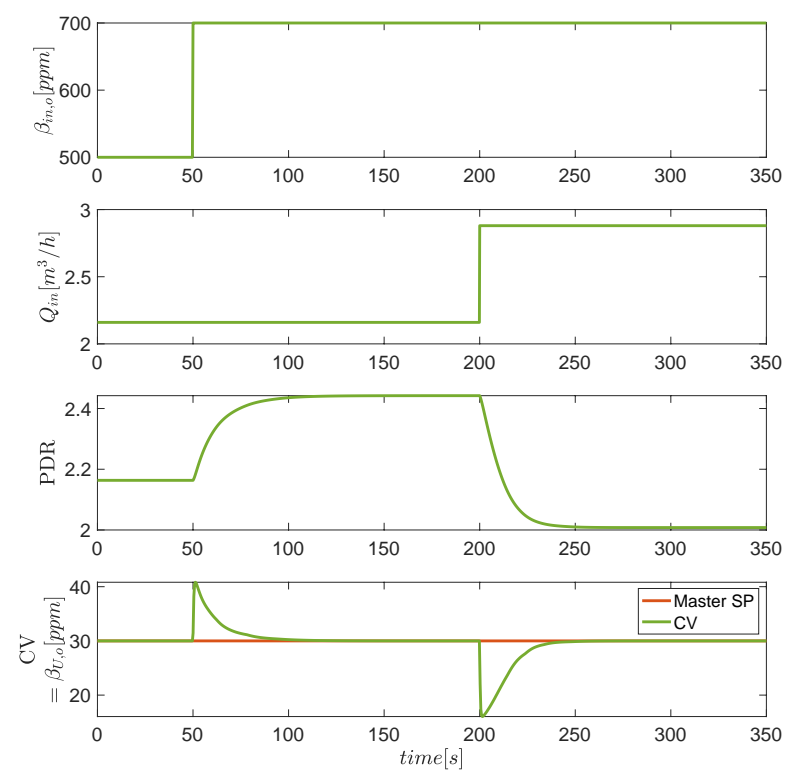

Figure 9: Simulation result showing the cascade control scheme. Here the inlet oil concentration is changed at $50 \mathrm{~s}$ and the inflow rate is changed at $200 \mathrm{~s}$.

\section{Model predictive control for hydrocyclones}

In Model Predictive Control (MPC), we use a process dynamic model in combination with an optimization method for determining the manipulated variables' values. These values are chosen such that the deviation between the predicted controlled variables values and their reference is minimized; the MPC determines the optimal input by simulating its internal model (Seborg et al., 2010). Here, we implement a nonlinear MPC that regulates the fraction of oil at the underflow at a given setpoint by manipulating the flowrate of the 
overflow by the use of the overflow control valve. In order to obtain a model suited for MPC applications, we rewrite the model of Section 2 into a state-space form:

$$
\begin{aligned}
& \dot{x}_{1}=\frac{1}{V_{O}}\left(Q_{s e p}-x_{1} K u-Q_{E x, o}\right) \\
& \dot{x}_{2}=\frac{1}{V_{F}}\left(Q_{i n, o}-Q_{s e p}-x_{2} Q_{U}+Q_{E x, o}\right),
\end{aligned}
$$

where the states $x_{1}$ and $x_{2}$ represent overflow oil fraction $\beta_{O, o}$ and, underflow oil fraction $\beta_{U, o}$. The control input $u$ (system manipulated variable) is the overflow valve opening and $K=C_{v 2} \sqrt{\frac{2\left(P_{2}-P_{O}\right)}{\rho_{O}}}$. In simulation, we assume $P_{2}$ to be constant during the integration interval.

The control problem can be formulated as:

$$
\begin{aligned}
& \min _{\boldsymbol{x}, u} \frac{1}{2} \int_{t_{c}}^{t_{c}+T_{p}}\left(\left(y(t)-y^{S P}\right)^{T} Q\left(y(t)-y^{S P}\right)+\right. \\
& \left.\dot{u}(t)^{T} R \dot{u}(t)\right) d t \\
& \dot{\boldsymbol{x}}(t)=f(\boldsymbol{x}(t), u(t)), \quad t \in\left[t_{c}, t_{c}+T_{p}\right] \\
& y(t)=\left[\begin{array}{ll}
0 & 1
\end{array}\right] \boldsymbol{x}(t), \quad t \in\left[t_{c}, t_{c}+T_{p}\right] \\
& \boldsymbol{x}\left(t_{c}\right)=\boldsymbol{x}_{0}, \\
& u_{\min } \leq u(t) \leq u_{\max }, \quad t \in\left[t_{c}, t_{c}+T_{p}\right] \\
& -\dot{u}_{\max } \leq \dot{u}(t) \leq \dot{u}_{\max }, \quad t \in\left[t_{c}, t_{c}+T_{m}\right] \\
& \dot{u}(t)=0 \quad t \in\left[t_{c}+T_{m}, t_{c}+T_{p}\right] \cup\left[t, t+T_{s}\right],
\end{aligned}
$$

where, $\boldsymbol{x}$ are the states, $u$ the control input, and $f$ the system model, all described in (8);y is the measured output (fraction of oil at the underflow) and $y^{S P}$ its setpoint; $\dot{u}$ is the control input change. The inequality constraints represent technical restrictions on the control inputs $u ; u_{\min }, u_{\max }$ are the input lower and upper bounds, and $\dot{u}_{\max }$ is the maximum input change in one sampling time $T_{s}$ (i.e $\left[t, t+T_{s}\right]$ ). $t_{c}$ is the current time when the optimization problem is called. The prediction horizon $T_{p}$ represents the control interval evaluated in the optimization problem, and the control horizon $T_{m}$ is the horizon in which the manipulated variable can be optimized. Note that, after the control horizon $T_{m}$, the inputs are kept constant by setting $\dot{u}=0$. The MPC is run every $T_{s}$ seconds. $Q$ is a matrix $n_{y} \times n_{y}$ that penalizes deviations of $y(t)$ from its setpoint $y^{S P}$, and $R$ is a matrix $n_{u} \times n_{u}$ for penalizing manipulated variable movements.

\subsection{Implementation}

The plant model and the MPC algorithm were implemented in MATLAB using the CasADi framework (Andersson et al., 2019). The plant model $f$ is integrated using CVodes from the Sundials suite in CasADi. A multiple shooting algorithm (Bock and Plitt, 1984) is used for computing the numerical solution of the optimal control problem in (9). This algorithm allows us to re-arrange the model dynamic equations in such a way that we can solve (9) using standard optimization nonlinear programming (NLP) solvers. Here we use IPOPT, which is an interior point NLP solver included in CasADi (Wächter and Biegler, 2006).

The MPC is implemented in a receding horizon framework, i.e. we compute the trajectory $\boldsymbol{u}^{\star}=$ $\left[u_{1}^{\star}, u_{2}^{\star}, \ldots, u_{T_{p}}^{\star}\right]^{T}$ and apply only the first control move $u_{1}^{\star}$. The next time step, the process is repeated. This strategy is used for compensating unexpected system disturbances and plant-model mismatch (Rawlings et al., 2017). In the simulation, the plant model and the controller have the same sampling time of $0.01 \mathrm{~s}$ and the MPC is run every $0.01 \mathrm{~s}$.

\subsection{Simulation results}

The simulation results with the model predictive controller are shown in Figure 10. We use the same scenario as in the previous case studies. We start the simulation with inflow rate of $2.2 \mathrm{~m}^{3} / \mathrm{h}$ and the inlet oil concentration of $500 \mathrm{ppm}$. Then, at $40 \mathrm{~s}$, the inlet oil concentration is increased to $700 \mathrm{ppm}$, while the outlet setpoint for the outlet oil concentration is kept at $30 \mathrm{ppm}$. We see that the MPC automatically adjusts the overflow valve opening $Z_{o}$ to reject the disturbance in the feed. Since we added a maximum input change constraint to account for the system inertia (constraint on $\dot{u}$ ), the $Z_{o}$ profile is smooth, which is important for practical implementation. Next, we increase the inflow rate to $2.6 \mathrm{~m}^{3} / \mathrm{h}$. The MPC is also able to track the setpoint of $\beta_{U, o}$ for this disturbance. Note that we assume full state feedback, i.e. the states (overflow and underflow oil fractions) are measured at every sampling time. The MPC parameters are given in Table 2.

\section{Comparing the control scheme alternatives}

We compare the three proposed approaches in terms of necessary measurements, as well as model and solver requirements. Note, we do not focus on performance criteria such as integral squared error, integral absolute error, etc. Since these are highly dependent on the tuning of the approach, poorly chosen parameters could influence the comparison. Therefore, we focus on qualitative criteria that will help the practitioner/engineer decide which strategy should be used based on the available resources. The characteristics of the three methods are summed up in Table 1. 

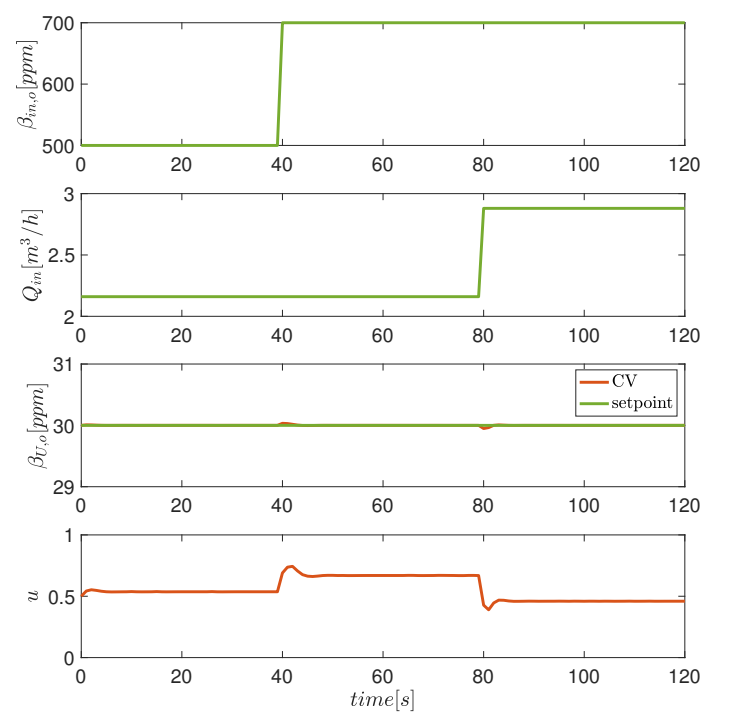

Figure 10: Simulation result of the MPC implementation. The controller first tracks the underflow oil fraction setpoint. Disturbances enter the system at $40 \mathrm{~s}$ and $80 \mathrm{~s}$, represented by a change in the inlet oil concentration and the inflow rate respectively. The controller rejects both the disturbance maintaining $\beta_{U, o}$ at its setpoint.

The implementation of the feed-forward approach requires online measurements of the inlet oilconcentration and inflow rate. They are used in combination with the feed-forward model to update the PDR setpoints according to the system disturbances. To obtain this model, we need historical data of the two previously mentioned measurements, as well as a specific values of the PDR setpoints. These values are chosen such that, given an inlet condition, the underflow concentration stays under a threshold, e.g., lower than $30 \mathrm{ppm}$. The setpoints can be obtained via simulations or based on process knowledge. Ideally, we need data from a large operation envelope to ensure that the feed-forward strategy works in different plant conditions. This feed-forward approach has the disadvantage that, in scenarios such as slugging where the inlet-oil concentration increases rapidly, the changes in the PDR setpoint aiming at improving separation can cause more water at the overflow outlet (oil-rich stream).

In comparison to the feed-forward approach, the feedback/cascade control requires measurement only of the underflow oil concentration. This method is a direct way of controlling the efficiency of hydrocyclones. The secondary PDR controller responds to changes in the inflow rates and maintains the PDR at a given setpoint. However, if a disturbance occurs in the inlet oil concentration and/or in the inlet oildroplet distribution, the separation efficiency of the hydrocyclone changes, and this will not be reflected in the PDR setpoint. Then, the primary controller can detect these disturbances by measuring the underflow oil concentration, and adjust the PDR setpoint to improve the efficiency. Note that there is an upper bound in the PDR beyond which the separation cannot be improved. In this case, if the primary controller tries to increase the PDR setpoint to maintain the separation efficiency, more water goes out through the overflow outlet. Hence, it is important to know the limitation of the system in use and deactivate the primary controller at the right time, which can turn out to be a major disadvantage of this approach.

This deficiency can be mitigated by the modelpredictive control approach. By using MPC, we can explicitly take into account operating constraint while dealing with the feed disturbances. Moreover, MPC has the potential to increase the separation regularity since it considers the interaction among multiple system variables, whereas PI controllers act based on single-input, single output relationships (Qin and Badgwell, 2003). The main disadvantage of the MPC formulation is that it requires a fairly accurate model. Also, in the model formulation proposed in this paper, we assume that underflow and overflow oil concentration are measured. It is not common to have an oil in water sensor at the overflow outlet of the hydrocyclones. As an alternative, we could use soft-sensors such as Kalman filters to estimate the overflow oil concentration. Another possibility of future work is to re-formulate the optimisation problem to minimize the underflow $\beta_{U, o}$ (instead of tracking a setpoint) and also maximize the total throughput of the hydrocyclones by adjusting the underflow valve.

\section{Conclusions}

In this paper, we propose three new control schemes for de-oiling hydrocyclones and study the effectiveness of these controllers when subjected to different plant disturbances. The schemes were designed in order to address issues with the standard hydrocyclone control strategy. Typically, a PDR controller is used to keep the underflow oil concentration at a given setpoint. However, if the inlet oil concentration and/or in the inlet oil-droplet distribution change, the PDR setpoint needs to be updated or the outlet purity requirements will not be met. The first scheme uses a feed-forward model to update the setpoint of a typical PDR controller. The second scheme is a feedback/cascade ap- 
Table 1: Comparison of the three approaches used for controlling the underflow oil fraction of a hydrocyclone.

\begin{tabular}{|c|c|c|c|c|}
\hline Approach & $\begin{array}{c}\text { Required } \\
\text { measurements }\end{array}$ & Model & $\begin{array}{l}\text { Optimization } \\
\text { solver }\end{array}$ & $\begin{array}{l}\text { Possible } \\
\text { disadvantages }\end{array}$ \\
\hline Feed-forward & $\begin{array}{l}\text { ○ Inlet oil fraction } \\
\text { ○ Inflow rate }\end{array}$ & $\begin{array}{l}\text { Feed-forward model to } \\
\text { predict PDR setpoint } \\
\text { changes }\end{array}$ & No & $\begin{array}{l}\circ \text { Fast inlet oscillations may } \\
\text { lead to high concentrations } \\
\text { of water at the } \\
\text { overflow outlet }\end{array}$ \\
\hline $\begin{array}{l}\text { Feed- } \\
\text { back/cascade }\end{array}$ & $\begin{array}{l}\text { o Underflow oil frac- } \\
\text { tion }\end{array}$ & No & No & $\begin{array}{l}\text { o Primary controller may } \\
\text { need to be deactivated } \\
\text { if upper bound in the PDR } \\
\text { is violated, which causes high } \\
\text { water concentration at the } \\
\text { overflow outlet }\end{array}$ \\
\hline $\begin{array}{l}\text { Model Pre- } \\
\text { dictive } \\
\text { Control }\end{array}$ & $\begin{array}{l}\text { - Overflow oil fraction } \\
\text { ○ Underflow oil fraction }\end{array}$ & $\begin{array}{l}\text { Yes. } \\
\text { State-space model of } \\
\text { Eq. (8) can be used }\end{array}$ & Yes & $\begin{array}{l}\text { o Fairly accurate model } \\
\text { needed } \\
\text { o Oil in water sensor is } \\
\text { necessary (or a soft sensor } \\
\text { needs to be developed). }\end{array}$ \\
\hline
\end{tabular}

Table 2: Tuning parameters

\begin{tabular}{|c|c|c|}
\hline Description & Variable & Value \\
\hline \multicolumn{3}{|c|}{ Feed-forward } \\
\hline PDR control prop. gain & $K_{c}$ & 0.67 \\
\hline PDR control int. gain & $\tau_{I}$ & 0.047 \\
\hline \multicolumn{3}{|c|}{ Feed-back/cascade } \\
\hline Primary control prop. gain & $K_{c}$ & 0.5 \\
\hline Primary control int. gain & $\tau_{I}$ & 0.5 \\
\hline Secondary control prop. gain & $K_{c}$ & -542.16 \\
\hline Secondary control int. gain & $\tau_{I}$ & 0.2598 \\
\hline \multicolumn{3}{|c|}{ Model Predictive Control } \\
\hline Setpoint weighting matrix & $Q$ & $5 \mathrm{e} 8$ \\
\hline Input weighting matrix & $R$ & 0.01 \\
\hline Prediction horizon & $T_{p}$ & 15 \\
\hline Control horizon & $T_{m}$ & 10 \\
\hline Input upper bounds & $u_{\max }$ & 0.01 \\
\hline Input lower bounds & $u_{\min }$ & 1 \\
\hline Max input change & $\dot{u}_{\max }$ & 0.5 \\
\hline
\end{tabular}

proach, where the primary controller takes measurements of oil concentration at the underflow outlet and updates the setpoint of the secondary controller (PDR controller) to maintain the underflow oil concentration at a required level. The third control scheme is a model predictive controller. Here, the control objective is to minimize the variation of the underflow oil concentration from a reference point while taking into account operating constraints, such as max water-in-oil concen- trations.

In the simulations, we see that all the control schemes meet the requirement to keep the underflow oil concentration below $30 \mathrm{ppm}$ other than briefly during transients. We also present guidelines for choosing the appropriate scheme based on the available resources at the industrial site (such as measurements, hardware and software at hand). As an extension of this work feed-forward and cascade control schemes are implemented at a newly constructed experimental rig at NTNU and result are being finalised.

\section{Acknowledgements}

This project is supported by the Norwegian Research Council, industrial partners and NTNU under the Subsea Production and Processing (SUBPRO) SFI program.

\section{References}

Andersson, J. A. E., Gillis, J., Horn, G., Rawlings, J. B., and Diehl, M. CasADi - A software framework for nonlinear optimization and optimal control. Mathematical Programming Computation, 2019. 11(1):1-36. doi:10.1007/s12532-018-0139-4.

Beyer, J., Bakke, T. H., Lichtenthaler, R., and Klungs $\varnothing y r$, J. Environmental effects of offshore produced water discharges evaluated for the barents sea. NIVA-rapport, 2019. 
Bock, H. G. and Plitt, K.-J. A multiple shooting algorithm for direct solution of optimal control problems. IFAC Proceedings Volumes, 1984. 17(2):1603-1608. doi:10.1016/S1474-6670(17)61205-9.

Bram, M. V., Hansen, L., Hansen, D. S., and Yang, Z. Hydrocyclone separation efficiency modeled by flow resistances and droplet trajectories. 3rd IFAC Workshop on Automatic Control in Offshore Oil and Gas Production, 2018. 51(8):132-137. doi:10.1016/j.ifacol.2018.06.367.

Bram, M. V., Jespersen, S., Hansen, D. S., and Yang, Z. Control-oriented modeling and experimental validation of a deoiling hydrocyclone system. Processes, 2020. 8(9):1010. doi:10.3390/pr8091010.

Das, T. and Jäschke, J. Modeling and control of an inline deoiling hydrocyclone. 3rd IFAC Workshop on Automatic Control in Offshore Oil and Gas Production OOGP, 2018. 51(8):138-143. doi:10.1016/j.ifacol.2018.06.368.

Durdevic, P., Pedersen, S., Bram, M., Hansen, D., Hassan, A., and Yang, Z. Control oriented modeling of a de-oiling hydrocyclone. 17th IFAC Symposium on System Identification SYSID, 2015. 48(28):291-296. doi:10.1016/j.ifacol.2015.12.141.

Durdevic, P. and Yang, Z. Application of hinf robust control on a scaled offshore oil and gas de-oiling facility. Energies, 2018. 11(2):287. doi:10.3390/en11020287.

Hansen, L., Durdevic, P., Jepsen, K. L., and Yang, Z. Plant-wide optimal control of an offshore de-oiling process using mpc technique. Ifac-papersonline, 2018. 51(8):144-150. doi:j.ifacol.2018.06.369.

Husveg, T., Rambeau, O., Drengstig, T., and Bilstad, T. Performance of a deoiling hydrocyclone during variable flow rates. Minerals Engineering, 2007. 20(4):368-379. doi:10.1016/j.mineng.2006.12.002.

MATLAB. Gaussian process regression models, https://se.mathworks.com/help/stats/gaussianprocess-regression-models.html. 2021. (accessed Sep , 2021).

Meldrum, N. Hydrocyclones: A solution to producedwater treatment. SPE Production Engineering, 1988. 3(04):669-676. doi:10.2118/16642-PA.

Orlowski, R., Euphemio, M. L. L., Euphemio, M. L., Andrade, C. A., Guedes, F., Tosta da Silva, L. C.,
Pestana, R. G., de Cerqueira, G., Lourenço, I., and Pivari, A. Marlim 3 phase subsea separation systemchallenges and solutions for the subsea separation station to cope with process requirements. In Offshore Technology Conference. Offshore Technology Conference, 2012. doi:10.4043/23552-MS.

Pereira, R. M., Campos, M. C. M. M. d., de Oliveira, D. A., de Souza, R. d. S. A., Filho, M. M. C., Orlowski, R., Duarte, D. G., Raposo, G. M., Lillebrekke, C., Ljungquist, D., Carvalho, A., and Fares, M. Ss: Marlim 3 phase subsea separation system: Controls design incorporating dynamic simulation work. paper presented at the offshore technology conference, houston, texas, usa, 30 april 3 may. Houston, Texas, USA, 2012. doi:10.4043/23564-MS.

Qin, S. J. and Badgwell, T. A. A survey of industrial model predictive control technology. Control engineering practice, 2003. 11(7):733-764. doi:10.1016/S0967-0661(02)00186-7.

Rawlings, J. B., Mayne, D. Q., and Diehl, M. Model predictive control: theory, computation, and design, volume 2. Nob Hill Publishing Madison, WI, 2017.

Seborg, D. E., Mellichamp, D. A., Edgar, T. F., and Doyle III, F. J. Process dynamics and control. third edition. John Wiley \& Sons, 2010.

Vallabhan, M. and Holden, C. Non-linear control algorithms for de-oiling hydrocyclones. In 2020 28th Mediterranean Conference on Control and Automation (MED). IEEE, pages 85-90, 2020. doi:10.1109/MED48518.2020.9183115.

Vallabhan K G, M., Holden, C., and Skogestad, S. A first-principles approach for control-oriented modeling of de-oiling hydrocyclones. Industrial \& Engineering Chemistry Research, 2020. 59(42):1893718950. doi:10.1021/acs.iecr.0c02859.

Wächter, A. and Biegler, L. T. On the implementation of an interior-point filter line-search algorithm for large-scale nonlinear programming. Mathematical programming, 2006. 106(1):25-57. doi:10.1007/s10107-004-0559-y.

Williams, C. K. and Rasmussen, C. E. Gaussian processes for machine learning, volume 2. MA:MIT press Cambridge, 2006. 